\title{
Spin-phonon coupling parameters from maximally localized Wannier functions and first-principles electronic structure: Single-crystal durene
}

\author{
Subhayan Roychoudhury and Stefano Sanvito \\ School of Physics and CRANN Institute, Trinity College, Dublin 2, Ireland
}

(Received 12 July 2018; published 17 September 2018)

\begin{abstract}
Spin-orbit interaction is an important vehicle for spin relaxation. At finite temperature lattice vibrations modulate the spin-orbit interaction and thus generate a mechanism for spin-phonon coupling, which needs to be incorporated in any quantitative analysis of spin transport. Starting from a density functional theory ab initio electronic structure, we calculate spin-phonon matrix elements over the basis of maximally localized Wannier functions. Such coupling terms form an effective Hamiltonian to be used to extract thermodynamic quantities, within a multiscale approach particularly suitable for organic crystals. The symmetry of the various matrix elements is analyzed by using the $\Gamma$-point phonon modes of a one-dimensional chain of $\mathrm{Pb}$ atoms. Then the method is employed to extract the spin-phonon coupling of solid durene, a high-mobility crystal organic semiconductor. Owing to the small masses of carbon and hydrogen spin orbit is weak in durene and so is the spin-phonon coupling. Most importantly, we demonstrate that the largest contribution to the spin-phonon interaction originates from Holstein-like phonons, namely, from internal molecular vibrations.
\end{abstract}

DOI: 10.1103/PhysRevB.98.125204

\section{INTRODUCTION}

In a nonmagnetic material the electrical resistance experienced by a charge carrier is independent of its spin. In contrast, when the material is magnetic, the resistance typically depends on the relative orientation of the carrier spin and the local magnetization [1]. This observation inspired the advent of the field of spin electronics, or spintronics [2], which concerns the injection, manipulation, and detection of spins in a solid-state environment. A prototype spintronic device, the spin valve [3], consists of two ferromagnetic layers sandwiching a nonmagnetic spacer [4], which can display a metallic $[5,6]$, insulating [7], or semiconducting $[8,9]$ electronic structure. The carriers, which are spin polarized by one ferromagnet, travel through the spacer to the other ferromagnet. If the spin direction is maintained during such a transfer, then the total resistance of the device will depend on the mutual orientation of the magnetization vectors of the two ferromagnets. It is then crucial to understand how the spin direction evolves during the motion of the carriers through the spacer and, in particular, to understand how this is preserved.

There are multiple possible sources of spin relaxation in a material, such as the presence of impurities, hyperfine interaction, and spin-orbit (SO) coupling. A theoretical description of all such phenomena is needed for an accurate evaluation of the quantities related to spin relaxation. The relative dominance of one interaction over the others is typically highly dependent on the specific material. In this work, we shall focus on SO interaction, more specifically, on the modulation of such interaction due to lattice vibrations. The spin of an electron interacts with the magnetic field generated by the relative motion of the nucleus about the electron, giving rise to SO interaction. At finite temperature the atoms of a solid vibrate with respect to their equilibrium positions with the amplitudes of such vibrations increasing with temperature.
Such vibrations, the phonons, change the potential felt by the electrons, including the component due to SO coupling [10]. This effectively generates a mechanism for spin-phonon coupling [11], which is key for the calculation of quantities related to spin relaxation in many systems. Thus, the term "spin-phonon coupling" has been used here to indicate only the modulation of the spin-orbit interaction originating from the ionic movement. This has to be distinguished from the term "spin-phonon coupling," which, in the study of multiferroics, is used to indicate the modulation of phonon frequencies due to changes in magnetic ordering [12-17]. Here we are interested in the opposite, namely, in the change in electronic structure brought by the vibrations, in particular for the case of organic crystals. Furthermore, it has to be noted that in organic compounds bearing permanent spin moments, such as molecular magnets crystals, there is a second mechanism for spin-phonon coupling, where the ions modulate the spinspin dipolar interaction. Such a mechanism is unlikely to be relevant for high-mobility organic crystals since they do not present localized moments.

Recent years have witnessed growing interest in exploring the possibility of using organic crystal semiconductors for electronic and spintronic applications [18-22]. This stems from the high degree of mechanical flexibility, the light weight and the ease of synthesis, and the patterning that characterize organic compounds. In these systems covalently bonded organic molecules are held together by weak van der Waals interactions. Due to the weak bonds between the individual molecules, vibrational motions are prominent in organic crystals, and the coupling of the vibrations to the charge carriers plays a crucial role [23] in the transport properties of such materials.

The presence of experimental evidence in support of different transport regimes [24-28] has generated a significant debate on whether the transport in organic crystals is dom- 
inated by delocalized bandlike transport, as in covalently bonded inorganic semiconductors, by localized hopping, or by a combination of both. The relative dominance of different transport-mechanisms could very well depend on the specific crystal and the experimental conditions, such as the temperature. Typically, in organic crystals the vibrational degrees of freedom are thought to introduce significant dynamical disorder $[29,30]$ and thereby to have paramount influence on the transport properties. Since the typical energies associated with lattice vibrations in organics are of the same order of magnitude of the electronic bandwidth, the coupling between carriers and phonons cannot be treated by perturbation theory. Thus, in general, formulating a complete theoretical framework for the description of transport in organic crystals is more challenging than that for covalently bonded inorganic semiconductors [23,31]. Even more complex is the situation concerning spin transport, for which the theoretical description often relies on parameters extracted from experiments [32] or on approximate spin Hamiltonians [33]

One viable option towards a complete ab initio description of spin transport consists of constructing a multiscale approach, where information about the electronic and vibrational properties calculated with first-principles techniques is mapped onto an effective Hamiltonian retaining only the relevant degrees of freedom. For instance, this is the strategy for constructing effective giant-spin Hamiltonians with spin-phonon coupling for the study of spin relaxation in molecular magnets [34,35]. The approach presented here instead consists of projecting the electronic structure over appropriately chosen maximally localized Wannier functions (MLWFs) [36], which effectively define a tight-binding (TB) Hamiltonian. In a previous work [37] we described a computationally convenient scheme for extracting the SO coupling matrix elements for MLWFs. Here we extend the method to the computation of the spin-phonon matrix elements. Our derived Hamiltonian can be readily used to compute spintransport quantities, such as the spin relaxation length. The effect of spin-phonon coupling on such quantities is expected to depend heavily on the material and the temperature.

This paper is organized as follows. In the next section we introduce our computational approach and describe the specific implementation used. Then we present our results. We analyze first the symmetry of the various matrix elements by considering the simple case of a linear atomic chain of $\mathrm{Pb}$ atoms. Then we move to the most complex case of the durene crystal, a popular high-mobility organic semiconductor. Finally, we conclude.

\section{METHOD}

Wannier functions, which form the basis functions of the proposed TB Hamiltonian, are essentially the weighted Fourier transforms of the Bloch states of a crystal. From a set of $N^{\prime}$ isolated Bloch states $\left\{\left|\psi_{m \mathbf{k}}\right\rangle\right\}$, which, for instance, can be the Kohn-Sham (KS) eigenstates of a density functional theory (DFT) calculation, one can obtain $N^{\prime}$ Wannier functions. The $n$th Wannier ket centered at the lattice site $\mathbf{R}$, $\left|w_{n} \mathbf{R}\right\rangle$, is found from the prescription

$$
\left|w_{n \mathbf{R}}\right\rangle=\frac{V}{(2 \pi)^{3}} \int_{\mathrm{BZ}}\left[\sum_{m=1}^{N} U_{m n}^{\mathbf{k}}\left|\psi_{m \mathbf{k}}\right\rangle\right] e^{-i \mathbf{k} \cdot \mathbf{R}} d \mathbf{k},
$$

where $V$ is the volume of the primitive cell, $\left|\psi_{m \mathbf{k}}\right\rangle$ is the $m$ th Bloch vector, and the integration is performed over the first Brillouin zone (BZ). Here $U^{\mathbf{k}}$ is a unitary operator that mixes the Bloch states. In order to fix the gauge choice brought by $U^{\mathrm{k}}$ one minimizes the spread of a Wannier function, which is defined as

$$
\Omega=\sum_{n}\left[\left\langle w_{n \mathbf{0}}\left|r^{2}\right| w_{n \mathbf{0}}\right\rangle-\left|\left\langle w_{n \mathbf{0}}|\mathbf{r}| w_{n \mathbf{0}}\right\rangle\right|^{2}\right] .
$$

Such a choice defines the so-called MLWFs [38]. We use the code WANNIER90 [39] to construct such MLWFs.

Before introducing the spin-phonon coupling, we shall briefly discuss electron-phonon coupling, which is known to play a crucial role in charge transport [40-43]. Since the TB Hamiltonian operator $\hat{H}$ depends on the ionic positions, the ionic motions give rise to changes in $\hat{H}$. In addition, since the MLWFs are constructed from the Bloch states, which themselves depend on the ionic coordinates, lattice vibrations result in a change in the MLWFs as well. Therefore, the change in the Hamiltonian matrix elements due to the ionic motion, namely, the on-site energies and hopping integrals, originates from the combined action of (1) the change in $\hat{H}$ and (2) the change in the MLWFs basis. Hence, in the MLWF TB picture the variation of the matrix element $\varepsilon_{n m}$ due to an ionic displacement is given by

$$
\Delta \varepsilon_{n m}=\left\langle w_{n}^{f}\left|\hat{H}^{f}\right| w_{m}^{f}\right\rangle-\left\langle w_{n}^{i}\left|\hat{H}^{i}\right| w_{m}^{i}\right\rangle,
$$

where $w_{m}^{i}\left(w_{m}^{f}\right)$ and $\hat{H}^{i}\left(\hat{H}^{f}\right)$ are the initial (final) MLWF and the Hamiltonian operator, respectively. Equation (3) describes the variation of on-site energy or a hopping integral depending on whether $\left|w_{m}\right\rangle$ and $\left|w_{n}\right\rangle$ are located on the same site or on different sites. Since any general lattice vibration can be expanded as a linear combination of normal modes, one is typically interested in calculating $\Delta \varepsilon_{n m}$ due to vibrations along the normal-mode coordinates. In order to quantify the rate of such change, we define the electron-phonon coupling parameter $g_{m n}^{\lambda}$ for the $\lambda$ th phonon mode as the rate of change $\Delta \varepsilon_{m n}$ of $\varepsilon_{m n}$ with respect to a displacement $\Delta Q_{\lambda}$ along such a normal mode, namely,

$$
g_{m n}^{\lambda}=\left.\frac{\partial \varepsilon_{m n}}{\partial Q}\right|_{Q \rightarrow Q+\Delta Q_{\lambda}} .
$$

Here $Q$ describes the system's geometry, so that $Q \rightarrow Q+$ $\Delta Q_{\lambda}$ indicates that the partial derivative is to be taken with respect to the atomic displacement along the phonon eigenvector corresponding to mode $\lambda$.

This coupling constant is fundamentally different from that defined in a conventional TB formulation. In that case the electron-phonon coupling is simply defined as

$$
\alpha_{n m}^{\lambda}=\left.\frac{\partial\left(\left\langle\phi_{n}^{i}\left|\hat{H}^{f}-\hat{H}^{i}\right| \phi_{m}^{i}\right\rangle\right)}{\partial Q}\right|_{Q \rightarrow Q+\Delta Q_{\lambda}},
$$

where $\left|\phi_{n}^{i}\right\rangle$ is the $n$th basis function before the motion. Note that, at variance with Eq. (3), which takes into account the changes in both the operator and the basis set, in Eq. (5) only the Hamiltonian operator is modified, and the matrix element is evaluated with respect to the basis set corresponding to the equilibrium structure. Depending on the context, either of the quantities $g$ and $\alpha$ can be important. Since, in the 
present case, we are interested in the modulation of the on-site (hopping) parameter associated with a site (set of sites), for the remainder of this paper, unless stated otherwise, electronphonon coupling will always refer to the first description, i.e., $g_{m n}^{\lambda}$ of Eq. (4).

All matrix elements, including those associated with the SO coupling, depend on the ionic coordinates. In a previous paper [37] we described a method to calculate the SO matrix elements associated with the MLWF basis $\left\langle w_{m \mathbf{R}}^{s_{1}}\left|\hat{V}_{\mathrm{SO}}\right| w_{n \mathbf{R}^{\prime}}^{s_{2}}\right\rangle$ from those computed over the spin-polarized Bloch states $\left\langle\psi_{m, \mathbf{k}}^{s_{1}}\left|\hat{V}_{\mathrm{SO}}\right| \psi_{n, \mathbf{k}^{\prime}}^{s_{2}}\right\rangle$ (the superscript denotes the magnetic spin quantum number). The presence of SO coupling changes the MLWFs [44]. However, note that here the MLWFs computed in the absence of SO coupling are used as basis functions since they span the entire relevant Hilbert space. The term $\left\langle\psi_{m, \mathbf{k}}^{s_{1}}\left|\hat{V}_{\mathrm{SO}}\right| \psi_{n, \mathbf{k}^{\prime}}^{s_{2}}\right\rangle$ can be, in principle, calculated from any DFT implementation that incorporates SO coupling. Our choice is the SIESTA code [45], which uses an on-site approximation [46] for the SO coupling and gives the SO elements in terms of a set of localized atomic orbitals $\left\{\left|\phi_{\mu, \mathbf{R}_{l}}^{s}\right\rangle\right\}$ [47]. Hence, the basic flowchart for such calculation follows the general prescription

$$
\left\langle\phi_{\mu, \mathbf{R}_{j}}^{s_{1}}\left|\hat{V}_{\mathrm{SO}}\right| \phi_{\nu, \mathbf{R}_{l}}^{s_{2}}\right\rangle \rightarrow\left\langle\psi_{m, \mathbf{k}}^{s_{1}}\left|\hat{V}_{\mathrm{SO}}\right| \psi_{n, \mathbf{k}^{\prime}}^{s_{2}}\right\rangle \rightarrow\left\langle w_{m \mathbf{R}}^{s_{1}}\left|\hat{V}_{\mathrm{SO}}\right| w_{n \mathbf{R}^{\prime}}^{s_{2}}\right\rangle
$$

namely, from the SO matrix elements calculated for the SIESTA local orbitals one computes those over the Bloch functions and then the ones over the MLWFs.

Once the matrix elements $\left\langle w_{m \mathbf{R}}^{s_{1}}\left|\hat{V}_{\text {SO }}\right| w_{n \mathbf{R}^{\prime}}^{s_{2}}\right\rangle$ are known, it is possible to determine the spin-phonon coupling by following a prescription similar to that used for computing the electronphonon coupling in Eq. (4),

$$
g_{m, n}^{s_{1} s_{2}(\lambda)}=\left.\frac{\partial \varepsilon_{(\mathrm{SO}) m n}^{s_{1} s_{2}}}{\partial Q}\right|_{Q \rightarrow Q+\Delta Q_{\lambda}},
$$

where $\varepsilon_{(\mathrm{SO}) m n}^{s_{1} s_{2}}$ is the SO matrix element between the MLWFs $\left|w_{m}^{s_{1}}\right\rangle$ and $\left|w_{n}^{s_{2}}\right\rangle, Q$ denotes the atomic positions, and $\Delta Q$ refers to an infinitesimal displacement of the coordinates along the $\lambda$ th phonon mode. As noted earlier, a change in atomic coordinates results in a change in the MLWFs, and such a change must be taken into account when calculating the difference in the SO elements $\Delta \varepsilon_{(\mathrm{SO}) m n}^{s_{1} s_{2}}$. We use the same symbol $g$ to denote both the electron-phonon and spin-phonon couplings since they can be distinguished by the presence or absence of the spin indices.

In practice, when calculating both the electron-phonon and spin-phonon couplings, each atom $i$ in the unit cell is infinitesimally displaced by $\Delta Q_{\lambda} \mathbf{e}_{\lambda}^{\mathbf{i}}$ along the direction of the corresponding phonon eigenvector $\mathbf{e}_{\lambda}^{\mathbf{i}}$. Then the electronphonon (spin-phonon) coupling is calculated as $\Delta \varepsilon_{m n} / \Delta Q_{\lambda}$ $\left(\Delta \varepsilon_{(\mathrm{SO}) m n}^{s_{1} s_{2}} / \Delta Q_{\lambda}\right)$, i.e., from finite differences. If $\Delta Q_{\lambda}$ is too large, then the harmonic approximation, which is the basis of this approach, breaks down. In contrast, if $\Delta Q_{\lambda}$ is too small, then the quantity will have a significant numerical error. Hence, for any system studied, one must evaluate the coupling term for a range of $\Delta Q_{\lambda}$ and, from a plot of coupling terms vs $\Delta Q_{\lambda}$, choose the most suitable value of $\Delta Q_{\lambda}$. It is important to note that the coupling terms so defined have the dimension of energy/length. This is consistent with the semiclassical

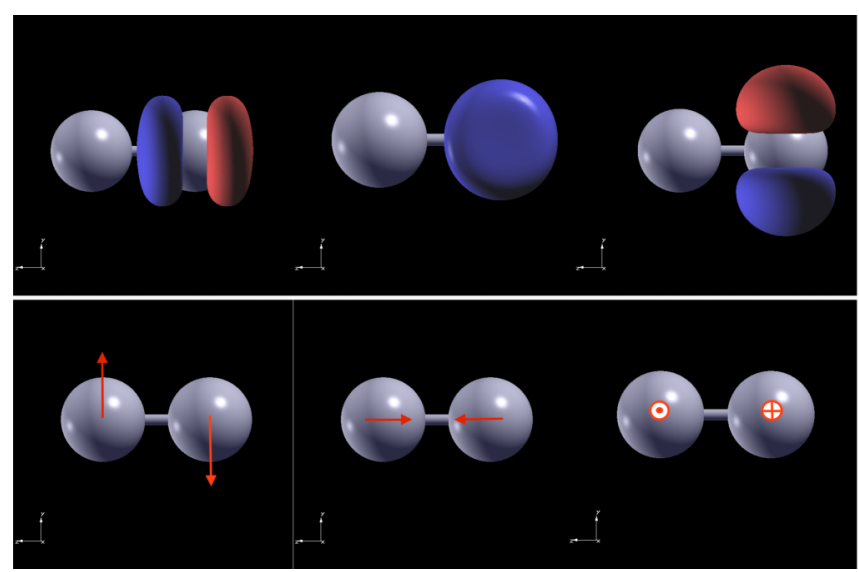

FIG. 1. The unit cell of the $\mathrm{Pb}$ chain containing two atoms. The figures in the top panel show isovalue plots of the three MLWFs (from left to right, $\left|w_{1, \mathbf{0}}\right\rangle,\left|w_{2, \mathbf{0}}\right\rangle$, and $\left|w_{3, \mathbf{0}}\right\rangle$ ) centered on the first atom. The bottom panel indicates the directions of the atomic motion corresponding to the three phonon modes (mode 1 , mode 2, and mode 3 , from left to right).

TB Hamiltonian used, for example, in Ref. [48] for treating transport in organic crystals with significant dynamic disorder. However, various other definitions and dimensions for the electron-phonon coupling can be found in the literature [43,49-51].

\section{RESULTS AND DISCUSSION}

\section{A. One-dimensional $\mathbf{P b}$ chain}

A linear chain of $\mathrm{Pb}$ atoms with a diatomic unit cell has six phonon modes for each wave vector q. For simplicity we restrict our calculations to the $\Gamma$ point, $\mathbf{q}=\mathbf{0}$, so that equivalent atoms in all unit cells have the same displacements with respect to their equilibrium positions. Since for the acoustic modes there is no relative displacement between the atoms of a unit cell, we are left with three optical modes of vibration, as shown in the bottom panel of Fig. 1. The electronic band structure of a diatomic $\mathrm{Pb}$ chain calculated with a single- $\zeta$ basis function is shown in Fig. 2. Note that two of the bands marked in red are composed mostly of $p$-orbital $\pi$ bonding and are doubly degenerate. Thus, as expected, the band structure contains eight bands in total. The MLWFs are constructed by omitting the lowest two bands (mostly made of $s$ orbitals) and retaining the remaining six bands. This gives us six MLWFs per unit cell, three centered on each atom. For each of the three modes, we evaluate the coupling matrix elements between the MLWFs of the same unit cell for a range of $\Delta Q_{\lambda}$. By analyzing these results we find that $\Delta Q_{\lambda}=0.03$ is an acceptable value for such fractional displacement.

The top panel of Fig. 1 shows the MLWFs corresponding to the first atom of the unit cell at the equilibrium geometry. From Fig. 1 one can see that $\left|w_{1, \mathbf{0}}\right\rangle,\left|w_{2, \mathbf{0}}\right\rangle$, and $\left|w_{3, \mathbf{0}}\right\rangle$ closely resemble the $p$ orbitals of the first atom, which we can denote arbitrarily (the definition of the axes is arbitrary) as $p_{z}$, $p_{x}$, and $p_{y}$, respectively. By symmetry, $\left|w_{4, \mathbf{0}}\right\rangle,\left|w_{5, \mathbf{0}}\right\rangle,\left|w_{6, \mathbf{0}}\right\rangle$ can be associated with the $p_{z}, p_{x}$, and $p_{y}$ orbitals located on the second atom. However, it is important to note that 


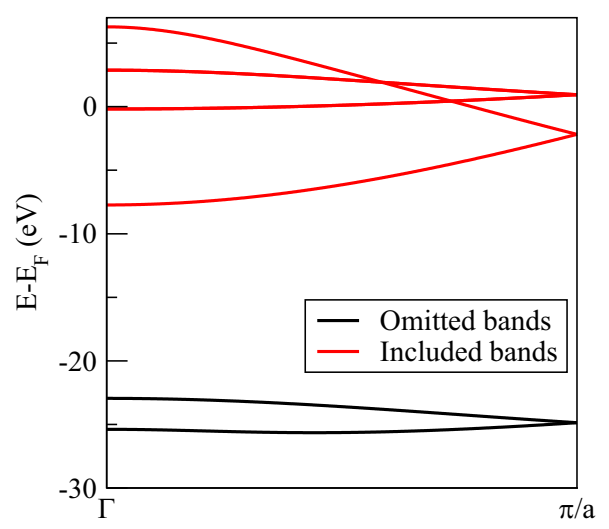

FIG. 2. Band structure of a diatomic $\mathrm{Pb}$ chain calculated with a minimal basis set in SIESTA. The black and red lines correspond to bands omitted from and included in the construction of the MLWFs, respectively.

such similarity between the MLWFs and the orbital angular momentum eigenstates does not mean that they are equivalent. In order to appreciate this point, note the following:

(1) $\left\langle w_{i, \mathbf{0}} \mid w_{j, \mathbf{0}}\right\rangle=0, \forall i \neq j$, but this is not necessarily true for $\left\langle p_{m, 1} \mid p_{n, 2}\right\rangle$, where $\left|p_{m, 1}\right\rangle$ and $\left|p_{n, 2}\right\rangle$ are orbital angular momentum eigenkets centered on the first and second atoms, respectively.

(2) When an atom is displaced from its equilibrium position, the $p$ orbitals (e.g., the basis orbitals of SIESTA) experience only a rigid shift but do not change in shape. In contrast, the MLWFs change in shape along with being displaced.

(3) Most importantly, in the on-site $\mathrm{SO}$ approximation used in SIESTA, the hopping term for SO coupling, i.e., the SO matrix element between two orbitals located on two different atoms, is always zero. As for the on-site term, the SO matrix element between two orbitals of the same atom is independent of the position of the other atom. Thus, the spin-phonon matrix elements are always zero when calculated with the on-site SO approximation over the SIESTA basis set. This is not the case for the MLWFs. Even when used in conjunction with an on-site SO approximation, the spin-phonon coupling

TABLE I. The nonvanishing electron-phonon coupling matrix elements for the $\Gamma$-point phonon modes of the $\mathrm{Pb}$ chain with a diatomic unit cell. Here $\left[w_{\mu} \mid w_{\nu}\right]$ denotes the electron-phonon coupling matrix element between the MLWFs $\left|w_{\mu}\right\rangle$ and $\left|w_{\nu}\right\rangle$. One must keep in mind that the matrix elements are real, and the remaining nonvanishing ones not reported here can be found from the relation $\left[w_{\mu} \mid w_{\nu}\right]=\left[w_{\nu} \mid w_{\mu}\right]$. See Fig. 1 for a diagram of the modes and the MLWFs.

\begin{tabular}{lcc}
\hline \hline Mode & Element & Value $(\mathrm{meV} / \AA)$ \\
\hline Mode 1 & {$\left[w_{3} \mid w_{4}\right]$} & -0.85 \\
Mode 2 & {$\left[w_{1} \mid w_{4}\right]$} & 4.03 \\
& {$\left[w_{2} \mid w_{5}\right]$} & -1.51 \\
& {$\left[w_{3} \mid w_{6}\right]$} & -1.51 \\
Mode 3 & {$\left[w_{2} \mid w_{4}\right]$} & -0.85 \\
\hline \hline
\end{tabular}

TABLE II. Spin-phonon coupling matrix elements for the $\Gamma$ point phonon modes of the $\mathrm{Pb}$ chain with a diatomic unit cell. Here $\left[w_{\mu}^{s_{1}} \mid w_{v}^{s_{2}}\right]$ denotes the complex spin-phonon coupling matrix element between the MLWFs $\left|w_{\mu}^{s_{1}}\right\rangle$ and $\left|w_{v}^{s_{2}}\right\rangle$. The remaining nonvanishing matrix elements can be found from the relations in Eq. (8). The phonon modes and the MLWFs are shown in Fig. 1.

\begin{tabular}{lcc}
\hline \hline Mode & Element & Value $(\mathrm{meV} / \AA)$ \\
\hline Mode 1 & {$\left[w_{1}^{\uparrow} \mid w_{5}^{\uparrow}\right]$} & $(0.0,-0.07)$ \\
& {$\left[w_{2}^{\uparrow} \mid w_{4}^{\uparrow}\right]$} & $(0.0,0.07)$ \\
& {$\left[w_{2}^{\uparrow} \mid w_{6}^{\downarrow}\right]$} & $(-0.19,0.0)$ \\
& {$\left[w_{3}^{\uparrow} \mid w_{5}^{\downarrow}\right]$} & $(0.19,0.0)$ \\
Mode 2 & {$\left[w_{1}^{\uparrow} \mid w_{5}^{\downarrow}\right]$} & $(0.05,0.0)$ \\
& {$\left[w_{2}^{\uparrow} \mid w_{4}^{\downarrow}\right]$} & $(-0.05,0.0)$ \\
& {$\left[w_{1}^{\uparrow} \mid w_{6}^{\downarrow}\right]$} & $(0.0,-0.05)$ \\
& {$\left[w_{3}^{\uparrow} \mid w_{4}^{\downarrow}\right]$} & $(0.0,0.05)$ \\
Mode 3 & {$\left[w_{1}^{\uparrow} \mid w_{6}^{\uparrow}\right]$} & $(0.0,0.07)$ \\
& {$\left[w_{3}^{\uparrow} \mid w_{4}^{\uparrow}\right]$} & $(0.0,-0.07)$ \\
& {$\left[w_{2}^{\uparrow} \mid w_{6}^{\downarrow}\right]$} & $(0.0,-0.19)$ \\
& {$\left[w_{3}^{\uparrow} \mid w_{5}^{\downarrow}\right]$} & $(0.00,0.19)$ \\
\hline \hline
\end{tabular}

is typically nonzero for a MLWF basis owing to the change in the basis functions upon ionic displacement.

Before calculating the spin-phonon coupling, let us take a brief look at the electron-phonon coupling matrix elements for the three phonon modes. The nonzero matrix elements are presented in Table I for each of the normal modes. It is interesting to note that the change in overlap between the associated " $p$ " orbitals due to the atomic displacements corresponding to the normal modes can be intuitively expected to have the same trend as the electron-phonon coupling matrix elements calculated with respect to the MLWFs (since the MLWFs closely resemble $p$ orbitals). For example, for an atomic motion along mode 3 (see Fig. 1), $\left\langle p_{y, 1} \mid p_{z, 2}\right\rangle$ must be zero since $\left|p_{z, 2}\right\rangle$ always has equal overlap with the positive and negative lobes of $\left|p_{y, 1}\right\rangle$. Keeping in mind that modes 1, 2, and 3 correspond, respectively, to a motion in the $y, z$, and $x$ directions, one can easily show that

$$
\begin{aligned}
\Delta\left\langle p_{z, 1} \mid p_{z, 2}\right\rangle_{\text {mode: } 2} & >\Delta\left\langle p_{z, 1} \mid p_{x, 2}\right\rangle_{\text {mode: } 3} \\
\Delta\left\langle p_{z, 1} \mid p_{x, 2}\right\rangle_{\text {mode: } 3} & =\Delta\left\langle p_{x, 1} \mid p_{z, 2}\right\rangle_{\text {mode }: 3} \\
& =\Delta\left\langle p_{y, 1} \mid p_{z, 2}\right\rangle_{\text {mode }: 1} \\
\Delta\left\langle p_{x, 1} \mid p_{y, 2}\right\rangle_{\text {mode: } 1} & =\Delta\left\langle p_{x, 1} \mid p_{z, 2}\right\rangle_{\text {mode } 2} \\
& =\Delta\left\langle p_{y, 1} \mid p_{z, 2}\right\rangle_{\text {mode }: 3} \\
& =0
\end{aligned}
$$

where $\Delta$ denotes a change in the overlap of the orbitals due to their corresponding atomic motion.

Now we proceed to present our results for the spin-phonon coupling. At variance with the electron-phonon coupling matrix elements, the spin-phonon ones are not necessarily real valued. For each of the three modes, the inequivalent nonzero spin-phonon coupling matrix elements are tabulated in Table II. We denote the spin-phonon matrix element between $\left|w_{\mu}^{s_{1}}\right\rangle$ and $\left|w_{v}^{s_{2}}\right\rangle$ as $\left[w_{\mu}^{s_{1}} \mid w_{v}^{s_{2}}\right]$. All other (equivalent) nonzero 
spin-phonon matrix elements can be found from those presented in Table II by using the following relations:

$$
\begin{aligned}
{\left[w_{\mu}^{\uparrow} \mid w_{\nu}^{\downarrow}\right] } & =-\left[w_{\mu}^{\downarrow} \mid w_{\nu}^{\uparrow}\right]^{*}, \\
{\left[w_{\mu}^{\uparrow} \mid w_{\nu}^{\downarrow}\right] } & =\left[w_{\nu}^{\downarrow} \mid w_{\mu}^{\uparrow}\right]^{*}, \\
\operatorname{Im}\left[w_{\mu}^{\uparrow} \mid w_{\nu}^{\uparrow}\right] & =-\operatorname{Im}\left[w_{\mu}^{\downarrow} \mid w_{\nu}^{\downarrow}\right] .
\end{aligned}
$$

Also, from the symmetry of the MLWFs, it is easy to show that

$$
\begin{aligned}
& {\left[w_{1}^{\uparrow} \mid w_{5}^{\uparrow}\right]_{\text {Mode1 }}=-\left[w_{2}^{\uparrow} \mid w_{4}^{\uparrow}\right]_{\text {Mode1 } 1},} \\
& {\left[w_{1}^{\uparrow} \mid w_{6}^{\uparrow}\right]_{\text {Mode3 }}=-\left[w_{3}^{\uparrow} \mid w_{4}^{\uparrow}\right]_{\text {Mode3 }} .}
\end{aligned}
$$

We have noted that in the on-site approximation, the spinphonon coupling (according to our definition) of the $\mathrm{Pb}$ chain should be zero when calculated over the SIESTA basis set. However, if such an on-site approximation is relaxed, one will be able to determine a number of analytical expressions for these coupling elements in terms of the change in orbital overlaps. It is interesting to note that the analytical expressions calculated in this way share many qualitative similarities with those presented in Table II. We summarize the findings of this section by noting that the spin-phonon couplings matrix elements corresponding to the two equivalent normal modes show the expected symmetry. We have also seen that the nonzero spin-phonon coupling matrix elements for mode 2 are, in general, smaller than those for the symmetry-equivalent modes 1 and 3 .

\section{B. Durene crystal}

Finally, we are in the position to discuss the spin-phonon coupling in a real organic crystal, namely, in durene. In an electron-phonon or spin-phonon coupling calculation, one needs to make sure that the construction of the MLWFs converges to a global minimum; otherwise, the various displaced geometries may correspond to different local minima, resulting in the description of a different energy landscape. Typically, a MLWF calculation with a dense $k$ mesh is likely to converge to a local minimum, while a calculation with a coarse $k$ mesh has a higher probability of giving the global minimum ( $\Gamma$-point calculation always converges to the global minimum). However, a coarse $k$ mesh translates to a small period for the Born-von Kármán boundary conditions, i.e., a poorer description of the crystal. In our calculation, we use a $4 \times 4 \times 4 k$ grid and construct the MLWFs from the top four valence bands. This enables the calculation to converge to a global minimum, identified by vanishing or negligible imaginary elements in the Hamiltonian matrix. In Fig. 3(a) we show a plot of the durene band structure (within a large energy window), and in Fig. 3(b), we show the band structure corresponding to the four bands used to construct MLWFs. These are plotted from the DFT SIESTA eigenvalues and by diagonalizing the tight-binding Hamiltonian constructed over the MLWFs.

Since the unit cell of durene contains two molecules, the four valence bands give us four MLWFs per unit cell, so that each molecule has two associated MLWFs. In Fig. 4 we show an isovalue plot of the four MLWFs corresponding to
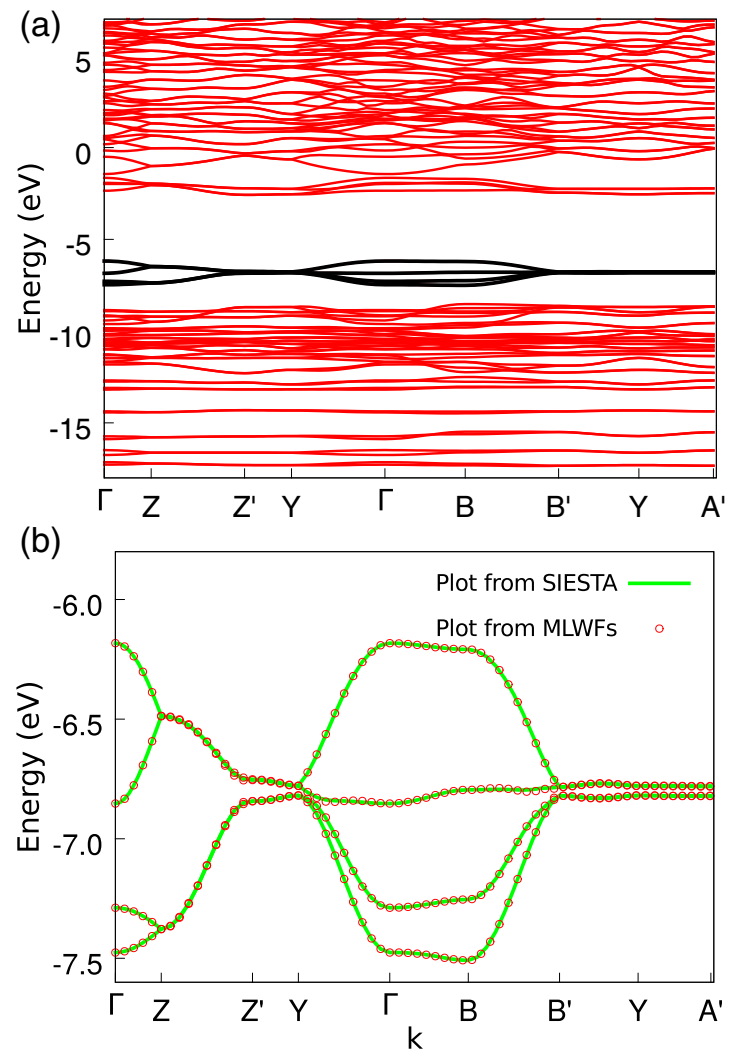

FIG. 3. Band structure of the durene crystal. (a) All the occupied and many unoccupied bands. MLWFs are constructed from the four highest occupied bands, which are plotted in black. (b) The magnified structure of these four bands plotted with SIESTA (green line) and obtained from the MLWFs computed with WANNIER90 (red circles).

$\mathbf{R}=\mathbf{0}$. We see that unlike $\left|w_{3, \mathbf{0}}\right\rangle$ and $\left|w_{4, \mathbf{0}}\right\rangle$, which are situated on the same molecule, $\left|w_{1, \mathbf{0}}\right\rangle$ and $\left|w_{2, \mathbf{0}}\right\rangle$ are on different but equivalent molecules displaced by a primitive lattice vector $\mathbf{a}_{2}$. Thus, $\left|w_{1, \mathbf{0}}\right\rangle$ and $\left|w_{2, \mathbf{R}^{\prime}}\right\rangle$ are on the same molecule for $\mathbf{R}^{\prime}=-\mathbf{a}_{2}$, where $\left\{\mathbf{a}_{1}, \mathbf{a}_{2}, \mathbf{a}_{3}\right\}$ is the set of primitive vectors. This means that for our tight-binding picture $\left\langle w_{1, \mathbf{0}}|\hat{H}| w_{2, \mathbf{0}}\right\rangle$

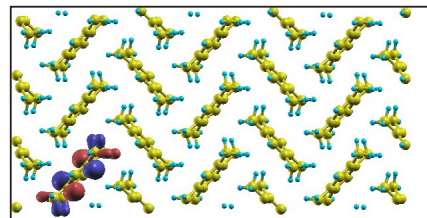

(a)

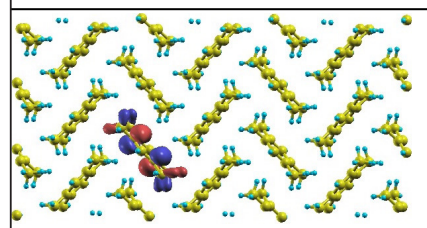

(c)

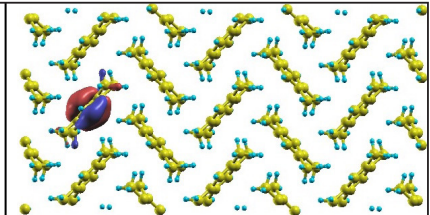

(b)

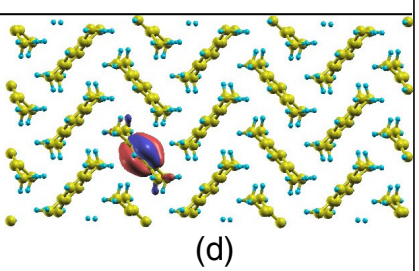

FIG. 4. Isovalue plots for MLWFs of the four topmost valence bands of a durene crystal: (a) $\left|w_{1, \mathbf{0}}\right\rangle$, (b) $\left|w_{2, \mathbf{0}}\right\rangle$, (c) $\left|w_{3, \mathbf{0}}\right\rangle$, and (d) $\left|w_{4,0}\right\rangle$. 
corresponds to a nonlocal (hopping) matrix element, whereas $\left\langle w_{1, \mathbf{0}}|\hat{H}| w_{2, \mathbf{R}^{\prime}}\right\rangle$ is a local (on-site) energy term. In the following, we shall calculate the electron-phonon and spin-phonon couplings corresponding to various modes of the durene crystal and compare (1) the relative contribution of the different modes and (2) for each mode, the relative contribution of the local and nonlocal terms.

Since the unit cell contains two molecules, each with 24 atoms (48 atoms in the unit cell), a $\Gamma$-point phonon calculation will give us 144 modes, with 141 being nontrivial. Among these, 12 will be predominantly intermolecular modes (3 translational and 9 rotational modes, where the molecules move rigidly with respect to each other), and the remaining ones will have a predominantly intramolecular nature. Here we shall consider only the phonon modes with an energy less than $75 \mathrm{meV}$, as the modes with higher energy are accessible only at high temperature [43]. Thus, we take into account 25 modes, of which the first 12 are intermolecular (these are lower in energy) and the rest are symmetry-inequivalent intramolecular ones [52].

In order to compare the contributions of the different phonon modes and of the local (Holstein-type) and nonlocal (Peierls-type) contributions, we calculate the following effective electron-phonon coupling parameters:

$$
G_{\lambda}^{\mathrm{L}}=\sum_{m, n}\left|g_{m n}^{\lambda}\right|^{2}
$$

where $m$ and $n$ are functions centered on the same molecule, and

$$
G_{\lambda}^{\mathrm{N}}=\sum_{m \neq n}\left|g_{m n}^{\lambda}\right|^{2}
$$

where $m$ and $n$ are on different molecules.

Here the superscripts $\mathrm{L}$ and $\mathrm{N}$ stand for local and nonlocal, respectively. A crucial point to be noted for treating bulk crystals is that in WANNIER90, the direct lattice points, where the MLWFs are calculated, are the lattice points of the WignerSeitz cell about the cell origin, $\mathbf{R}=\mathbf{0}$. Typically, one should expect the number of such lattice points to be the same as the number of $k$ points in reciprocal space. However, in a threedimensional crystal it is possible to have lattice points which are equidistant from the $\mathbf{R}=\mathbf{0}$ cell and (say) $n$ other cells. This means that such a lattice point is shared by Wigner-Seitz cells of $n+1$ cells. In this case, this degenerate lattice point is taken into consideration by WANNIER 90 , but a degeneracy weight of $1 /(n+1)$ is associated with it. Consequently, in further calculations (such as the band structure interpolation), its contribution carries a factor of $1 /(n+1)$. Keeping this in mind, we multiply the contributions from the MLWFs of degenerate direct lattice points by their corresponding weighting factors. Figure 5 shows a histogram of the $G_{\lambda}$ terms as a function of the phonon energy. It must be kept in mind that the coupling matrix elements are strongly dependent on the MLWFs. Therefore, constructing Wannier functions from a different set of Bloch states can, in principle, result in different values of $G_{\lambda}$. We see that in our case, most of the modes with high $G_{\lambda}\left(=G_{\lambda}^{\mathrm{L}}+G_{\lambda}^{\mathrm{N}}\right)$ are located at high phonon energies. Also, the electron-phonon couplings for modes with lower $G_{\lambda}$ are dominated by the nonlocal contributions, while those with higher $G_{\lambda}$ are dominated by local contributions.

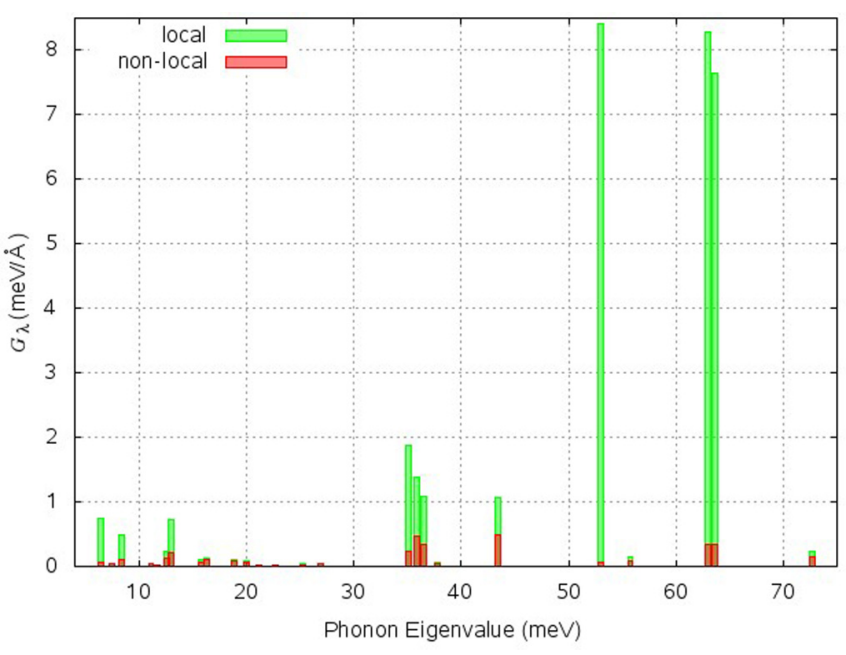

FIG. 5. Histogram of the effective electron-phonon coupling as a function of the phonon energy. The local and nonlocal contributions are denoted by green and red bars, respectively.

Concerning the spin-phonon coupling, we can define spindependent $G_{\lambda}$ terms, namely, the effective spin-phonon couplings,

$$
G_{\lambda}^{\mathrm{L}\left(\mathrm{s}_{1} \mathrm{~s}_{2}\right)}=\sum_{m, n}\left|g_{m n}^{s_{1} s_{2}(\lambda)}\right|^{2}
$$

where $m$ and $n$ are on same molecule, and

$$
G_{\lambda}^{\mathrm{N}\left(\mathrm{s}_{1} \mathrm{~s}_{2}\right)}=\sum_{m \neq n}\left|g_{m n}^{s_{1} s_{2}(\lambda)}\right|^{2},
$$

where $m$ and $n$ are on different molecules.

In Fig. 6, we plot these effective spin-phonon coupling terms, and we break down the local and nonlocal contributions. The top and bottom panels correspond to the $\left(s_{1}=\uparrow, s_{2}=\uparrow\right)$ and $\left(s_{1}=\uparrow, s_{2}=\downarrow\right)$ cases, respectively. As expected, the spin-phonon coupling terms are extremely small (about four orders of magnitude smaller than those of the $\mathrm{Pb}$ chain), owing to the small atomic masses in the crystal (the SO coupling is small). As in the case of the electronphonon interaction, the effective spin-phonon coupling terms are dominated by nonlocal contributions for low $G_{\lambda}^{\left(s_{1} S_{2}\right)}=$ $G_{\lambda}^{\mathrm{L}\left(\mathrm{s}_{1} s_{2}\right)}+G_{\lambda}^{\mathrm{N}\left(\mathrm{s}_{1} s_{2}\right)}$ and by local contributions for high $G_{\lambda}$. We also see that the spin-phonon coupling (for the same spin, as well as for different spins) is very small for the first few modes, which represent intermolecular motions. This is fully consistent with the short-range nature of SO coupling. An important message emerging from these results is that phonon modes with high effective electron-phonon coupling do not necessarily have high effective spin-phonon coupling and vice versa. This means that the knowledge of the phonon spectrum says little a priori about the spin-phonon coupling, so that any quantitative theory of spin relaxation cannot proceed unless a detailed analysis along the lines outlined here is performed.

In conclusion, we have discovered that both the electronphonon and spin-phonon coupling constants are, in general, dominated by the local modes, as expected from the shortrange nature of the SO coupling. However, modes with very small effective coupling tend to have a larger relative 

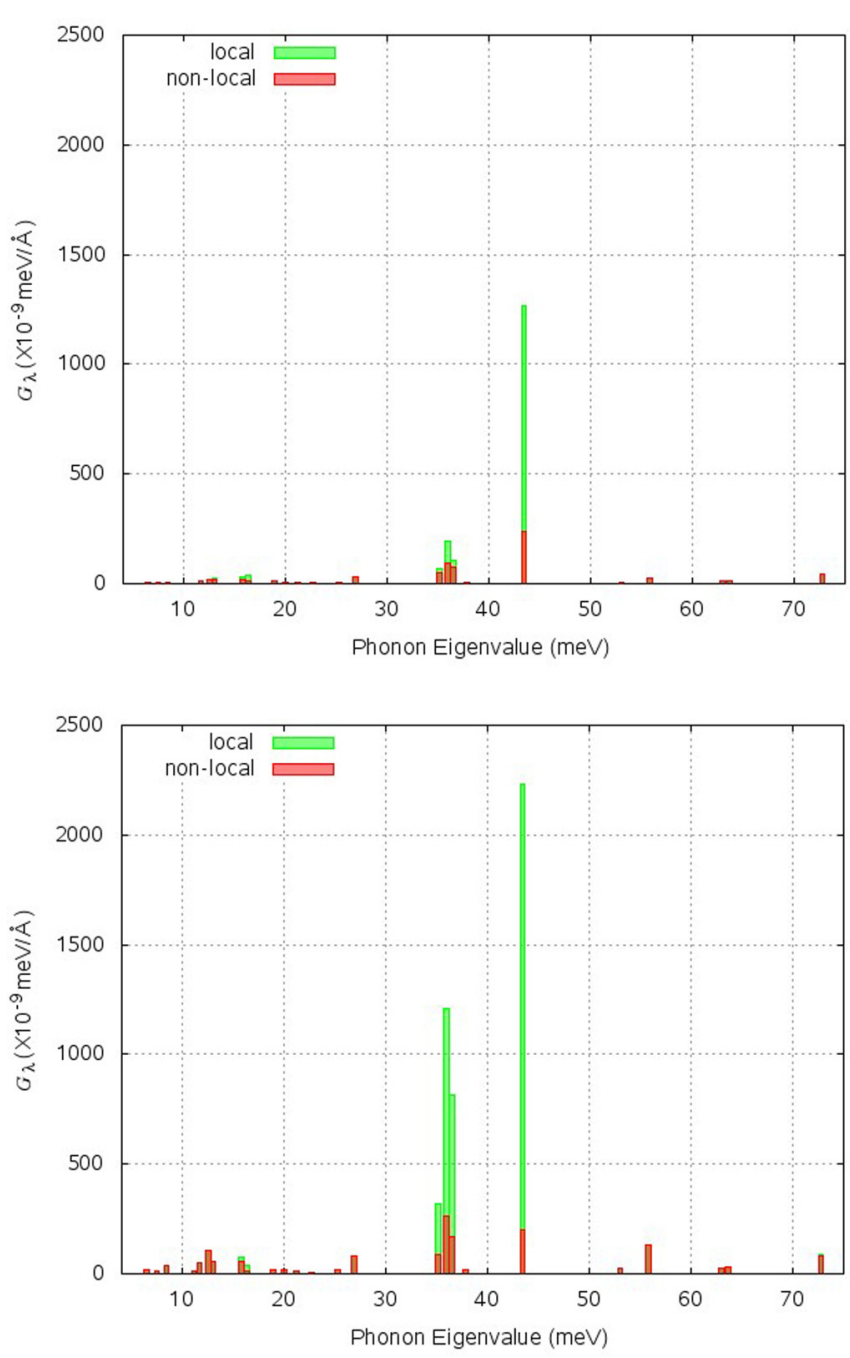

FIG. 6. Histogram of the effective spin-phonon coupling parameters $G_{\lambda}^{\left(\mathrm{s}_{1} \mathrm{~s}_{2}\right)}$ as a function of the phonon energy. The top panel corresponds to the $s_{1}=s_{2}$ case (same spins), while the bottom panel corresponds to $s_{1} \neq s_{2}$ (different spins). The local and nonlocal contributions are denoted by green and red bars, respectively.

contribution arising from nonlocal modes. No apparent correlation can be found between the effective coupling constants pertaining to various phonon modes for the electron-phonon coupling and those for the spin-phonon coupling.

\section{CONCLUSION}

Based on our previous work concerning the calculation of the SO matrix elements with respect to MLWFs basis sets, we have presented calculations of the spin-phonon coupling matrix elements of periodic systems. Although a quantity of mostly computational interest, such coupling terms can be incorporated within a multiscale approach for constructing a model Hamiltonian, which, in turn, can be solved to find quantities of practical and technological importance such as the spin-relaxation length/time. We note that, in order to be useful in such a multiscale approach, the electron-phonon and spin-phonon couplings are not to be calculated in terms of a fixed set of MLWFs. Instead, one must take into account the change in the MLWFs as a result of the ionic motions. The coupling matrix elements for a given phonon mode are calculated by displacing atoms from the ground-state geometry along that phonon eigenvector and by taking finite differences. For phonon modes at the $\Gamma$ point, we have calculated the electron-phonon and spin-phonon coupling elements of a onedimensional chain of $\mathrm{Pb}$ atoms with two atoms per unit cell and of a bulk durene crystal. The latter is a widely studied and well-known organic semiconductor. The spin-phonon coupling matrix elements of the $\mathrm{Pb}$ chain obey the expected symmetry relations. For durene we have observed that, in general, the spin-phonon coupling is dominated by local contributions (Holstein modes), although for phonon modes with a small net effective coupling, the nonlocal part seems to dominate. Our calculations of spin-phonon coupling matrix elements are expected to be valuable in the construction of an effective Hamiltonian to compute transport-related quantities. This is particularly welcome in the case of organic crystals, where $a b$ initio computation of transport properties is a challenging task.

\section{ACKNOWLEDGMENTS}

This work is supported by the European Research Council, Quest project. Computational resources were provided by the supercomputer facilities at the Trinity Center for High Performance Computing (TCHPC) and at the DJEI/DES/SFI/HEA Irish Center for High End Computing (ICHEC). We thank C. Motta for providing the $\Gamma$-point phonon eigenvalues and eigenvectors of durene crystal used in Ref. [43].
[1] N. F. Mott, Proc. R. Soc. A 153, 699 (1936).

[2] S. A. Wolf, D. D. Awschalom, R. A. Buhrman, J. M. Daughton, S. von Molnár, M. L. Roukes, A. Y. Chtchelkanova, and D. M. Treger, Science 294, 1488 (2001).

[3] B. Dieny, V. S. Speriosu, S. S. P. Parkin, B. A. Gurney, D. R. Wilhoit, and D. Mauri, Phys. Rev. B 43, 1297 (1991).

[4] S. Sanvito, Nat. Mater. 10, 484 (2011).

[5] M. N. Baibich, J. M. Broto, A. Fert, F. Nguyen Van Dau, F. Petroff, P. Etienne, G. Creuzet, A. Friederich, and J. Chazelas, Phys. Rev. Lett. 61, 2472 (1988).

[6] G. Binasch, P. Grünberg, F. Saurenbach, and W. Zinn, Phys. Rev. B 39, 4828 (1989).
[7] J. S. Moodera, L. R. Kinder, T. M. Wong, and R. Meservey, Phys. Rev. Lett. 74, 3273 (1995).

[8] J. Fabian, A. Matos-Abiague, C. Ertler, P. Stano, and I. Zutic, Acta Phys. Slovaca 57, 565 (2007).

[9] D. D. Awschalom and M. E. Flatté, Nat. Phys. 3, 153 (2007).

[10] F. P. Marin and H. Suhl, Phys. Rev. Lett. 63, 442 (1989).

[11] H. Ochoa, A. H. Castro Neto, V. I. Fal'ko, and F. Guinea, Phys. Rev. B 86, 245411 (2012).

[12] C. J. Fennie and K. M. Rabe, Phys. Rev. Lett. 96, 205505 (2006).

[13] T. Birol and C. J. Fennie, Phys. Rev. B 88, 094103 (2013).

[14] J. H. Lee and K. M. Rabe, Phys. Rev. B 84, 104440 (2011). 
[15] J. Hong, A. Stroppa, J. Íñiguez, S. Picozzi, and D. Vanderbilt, Phys. Rev. B 85, 054417 (2012).

[16] T. Birol, N. A. Benedek, H. Das, A. L. Wysocki, A. T. Mulder, B. M. Abbett, E. H. Smith, S. Ghosh, and C. J. Fennie, Curr. Opin. Solid State Mater. Sci. 16, 227 (2012).

[17] M. Mochizuki, N. Furukawa, and N. Nagaosa, Phys. Rev. B 84, 144409 (2011).

[18] G. Malliaras and R. Friend, Phys. Today 58(5), 53 (2005).

[19] Nat. Mater. 12, 591 (2013).

[20] S. R. Forrest, Nature (London) 428, 911 (2004).

[21] S. Sanvito, Chem. Soc. Rev. 40, 3336 (2011).

[22] S. Wang, A. Wittmann, K. Kang, S. Schott, G. Schweicher, R. D. Pietro, J. Wunderlich, D. Venkateshvaran, M. Cubukcu, and H. Sirringhaus, in 2017 IEEE International Magnetics Conference (INTERMAG) (IEEE, Piscataway, NJ, 2017), p. 1.

[23] A. Troisi, Chem. Soc. Rev. 40, 2347 (2011).

[24] C. Dimitrakopoulos and P. Malenfant, Adv. Mater. 14, 99 (2002).

[25] O. D. Jurchescu, J. Baas, and T. T. M. Palstra, Appl. Phys. Lett. 84, 3061 (2004).

[26] O. Ostroverkhova, D. G. Cooke, F. A. Hegmann, J. E. Anthony, V. Podzorov, M. E. Gershenson, O. D. Jurchescu, and T. T. M. Palstra, Appl. Phys. Lett. 88, 162101 (2006).

[27] K. Marumoto, S.-i. Kuroda, T. Takenobu, and Y. Iwasa, Phys. Rev. Lett. 97, 256603 (2006).

[28] T. Sakanoue and H. Sirringhaus, Nat. Mater. 9, 736 (2010).

[29] A. Troisi and G. Orlandi, J. Phys. Chem. A 110, 4065 (2006).

[30] A. Troisi, D. L. Cheung, and D. Andrienko, Phys. Rev. Lett. 102, 116602 (2009).

[31] K. Hannewald and P. A. Bobbert, Phys. Rev. B 69, 075212 (2004).

[32] P. A. Bobbert, W. Wagemans, F. W. A. van Oost, B. Koopmans, and M. Wohlgenannt, Phys. Rev. Lett. 102, 156604 (2009).

[33] S. Bhattacharya, A. Akande, and S. Sanvito, Chem. Commun. 50, 6626 (2014).

[34] A. Lunghi, F. Totti, R. Sessoli, and S. Sanvito, Nat. Commun. 8, 14620 (2017).
[35] A. Lunghi, F. Totti, S. Sanvito, and R. Sessoli, Chem. Sci. 8, 6051 (2017).

[36] N. Marzari, A. A. Mostofi, J. R. Yates, I. Souza, and D. Vanderbilt, Rev. Mod. Phys. 84, 1419 (2012).

[37] S. Roychoudhury and S. Sanvito, Phys. Rev. B 95, 085126 (2017).

[38] N. Marzari and D. Vanderbilt, Phys. Rev. B 56, 12847 (1997).

[39] A. A. Mostofi, J. R. Yates, Y.-S. Lee, I. Souza, D. Vanderbilt, and N. Marzari, Comput. Phys. Commun. 178, 685 (2008).

[40] F. Ortmann, K. Hannewald, and F. Bechstedt, Phys. Status Solidi B 245, 825 (2008).

[41] F. Ortmann, K. Hannewald, and F. Bechstedt, Appl. Phys. Lett. 93, 222105 (2008).

[42] H. Ishii, N. Kobayashi, and K. Hirose, Phys. Rev. B 95, 035433 (2017).

[43] C. Motta and S. Sanvito, J. Chem. Theory Comput. 10, 4624 (2014).

[44] F. Freimuth, Y. Mokrousov, D. Wortmann, S. Heinze, and S. Blügel, Phys. Rev. B 78, 035120 (2008).

[45] J. M. Soler, E. Artacho, J. D. Gale, A. Garcìa, J. Junquera, P. Ordejon, and D. Sanchez-Portal, J. Phys. Condens. Matter 14, 2745 (2002).

[46] In the on-site approximation a SO coupling matrix element is neglected unless both orbitals and the SO pseudopotential are on the same atom.

[47] L. Fernández-Seivane, M. A. Oliveira, S. Sanvito, and J. Ferrer, J. Phys. Condens. Matter 18, 7999 (2006).

[48] A. Troisi and G. Orlandi, Phys. Rev. Lett. 96, 086601 (2006).

[49] K. Kaasbjerg, K. S. Thygesen, and K. W. Jacobsen, Phys. Rev. B 85, 115317 (2012).

[50] A. Girlando, L. Grisanti, M. Masino, I. Bilotti, A. Brillante, R. G. Della Valle, and E. Venuti, Phys. Rev. B 82, 035208 (2010).

[51] V. Coropceanu, J. Cornil, D. A. da Silva Filho, Y. Olivier, R. Silbey, and J.-L. Brédas, Chem. Rev. 107, 926 (2007).

[52] The phonon spectrum is calculated with FHI-AIMS. Calculation courtesy of Dr. Carlo Motta. 